Pacific Journal of Mathematic 


\section{DIRECTED GRAPHS AS UNIONS OF PARTIAL ORDERS}

\section{Peter C. Fishburn ANd Joel H. Spencer}

The index of an irreflexive binary relation $R$ is the smallest cardinal number $\sigma(R)$ such that $R$ equals the union of $\sigma(R)$ partial orders. With $s(n)$ the largest index for an $R$ defined on $n$ points, it is shown that $s(n) / \log _{2} n \rightarrow 1$ as $n \rightarrow \infty$. The index function is examined for symmetric $R$ 's and almost transitive $R$ 's, and a characterization for $\sigma(R) \leqq 2$ is presented. It is shown also that

$$
\text { inf }\{n: s(n)>3\} \leqq 13 \text {, }
$$

but the exact value of $\inf \{n: s(n)>3\}$ is presently unknown.

1. Introduction. A binary relation on a set $X$ is a subset of ordered pairs $x y$ in $X \times X$. A directed graph (hereafter digraph ${ }^{1}$ ) $G=(X, R)$ is a nonempty set $X$ and an irrefiexive $(x x \notin R)$ binary relation $R$ on $X$. If $\phi \subset Y \subseteq X$ then $G \mid Y$ is the digraph obtained from $G=(X, R)$ by deleting all points in $X-Y$.

A partial order $P$ on $X$ is an irreflexive and transitive $(x y \in P \&$ $y z \in P \Rightarrow x z \in P$ ) binary relation on $X$. A digraph $G=(X, R)$ is resolved by a set of partial orders on $X$ if and only if $R$ equals the union of the partial orders in the set. Since $\{x y\}$ is a partial order when $x y \in R$, every $G$ is resolved by some set of partial orders.

The index ${ }^{2}$ of a digraph $G=(X, R)$ is the smallest cardinal number $\sigma(R)$ such that $R$ is resolved by $\sigma(R)$ partial orders on $X$. Clearly $\sigma(R)=1$ if and only if $R$ is a partial order. $\sigma(\{a b, b a\})=2$, and $\sigma(R)=3$ for the cyclic triangle $R=\{a b, b c, c a\}$. The smallest $X$ that we know of that admits an $R$ with $\sigma(R)=4$ has 13 points. (See Figure 1.) In connection with a later characterization of $\sigma \leqq 2$ we present an $R$ with $\sigma(R)=2$ where $R$ cannot be the union of two disjoint partial orders.

Our definition of $\sigma(R)$ is motivated by Dushnik and Miller's definition [2] of the dimension of a partial order $P$ on $X$ as the smallest cardinal number $D(P)$ such that $P$ equals the intersection of $D(P)$ linear orders on $X$. A linear order $L$ on $X$ is a complete $(x \neq y \Rightarrow x y \in L$ or $y x \in L)$ partial order, and a chain in $X$ is a linear

${ }^{1} \mathrm{We}$ shall sometimes refer to a binary relation as a digraph, omitting explicit mention of the set on which the relation is defined.

2 It is tempting to use "dimension" instead of "index," but since the former term is used for a number of other concepts in the theory of binary relations we favor the latter here. It would be proper to write $\sigma(G)$ instead of $\sigma(R)$, but since $\sigma(R)=\sigma\left(R^{\prime}\right)$ if $R$ is isomorphic to $R^{\prime}$ the specific omission of $X$ will cause no problems. 
order on a subset of $X$. A number of facts about $D(P)$ are summarized in [1], which gives other references.

This paper examines the index function $\sigma$ for digraphs. The next section focuses on large values for $\sigma(R)$. Our first theorem, based on a theorem in Folkman [4], shows that $\sigma(R)$ can be arbitrarily large for both symmetric $(x y \in R \Rightarrow y x \in R)$ and asymmetric $(x y \in R \Rightarrow$ $y x \notin R$ ) digraphs. The second theorem examines the behavior of $\sigma$ in the following way. Let

$$
s(n)=\sup \{\sigma(R): R \text { is an irreflexive binary relation on } n \text { points }\},
$$

the largest $\sigma$ for a digraph with $n$ points. When $u$ is a real-valued function on $\{1,2, \cdots\}$ and $u(n)$ remains bounded as $n$ gets large, we write $u=0(1)$ according to popular convention. Theorem 2 states that

$$
\log _{2} n-\frac{1}{2} \log _{2} \log _{2} n+0(1) \geqq s(n) \geqq \log _{2} n-\frac{3}{2} \log _{2} \log _{2} n-0(1) .
$$

This gives another proof that $\sigma$ can be arbitrarily large, and shows that $s(n) / \log _{2}(n)$ approaches 1 as $n$ gets large.

The rest of the paper is mostly concerned with small values of $\sigma$. Section 3 presents an $(X, R)$ with $|X|=13$ and $\sigma(R)=4$. We do not presently know the smallest $X$ that admits an $R$ with $\sigma(R)=4$.

Symmetric digraphs $(X, S)$ are examined in $\S 4$, where we give a necessary and sufficient condition for $\sigma(S) \leqq 2$. Suppose that $P$ is a partial order on $X$ and

$$
S=\{x y: x y \in X \times X \& x \neq y \& x y \notin P \& y x \notin P\} .
$$

Then $S$ is a symmetric digraph. We note that when $S$ is defined in this way, then $D(P) \leqq 2$ if and only if $\sigma(S) \leqq 2$, and

$$
D(P) \leqq n \Longrightarrow \sigma(S) \leqq 2(n-1) .
$$

The question of whether $\sigma(S) \leqq n \Rightarrow D(P) \leqq f(n)$ for some function $f$ is presently open.

A binary relation $R$ is almost transitive ${ }^{3}$ if and only if ( $a b \in R$ $\& b c \in R \& a \neq c) \Rightarrow a c \in R$. Section 5 proves that $\sigma(R) \leqq 2$ when $R$ is an almost transitive digraph.

Section 6 then gives a general characterization of $\sigma(R) \leqq 2$ that is stated in terms of a partition of the subset of $R$ whose elements

${ }^{3}$ Harary, Norman and Cartwright [7, p. 7] call this transitivity, but we use the modifier to distinguish it from the more common use of "transitivity" in which $a, b$ and $\mathrm{c}$ do not have to be distinct. 
are involved in nontransitive adjacent pairs such as $x y, y z \in R \&$ $x z \notin R$.

\section{Digraphs with large indices.}

THEOREM 1. If $n$ is a positive integer then there are asymmetric and symmetric digraphs whose indices exceed $n$.

Our proof is based on a specialization of Theorem 2 in Folkman [4]. A graph $(X, E)$ is a nonempty set $X$ and a set $E$ of unordered pairs $\{x, y\}$ with $x, y \in X$ and $x \neq y$. A triangle of $(X, E)$ is a set $\{\{a, b\},\{b, c\},\{a, c\}\} \subseteq E$. A partition of $X$ is a set of mutually disjoint subsets of $X$ whose union equals $X$.

Lemma 1 (Folkman). Let $m$ be a positive integer. Then there is a graph $(X, E)$ that includes no triangles, and every partition $\left\{C_{1}, \cdots, C_{k}\right\}$ of $X$ with $k \leqq m$ contains $a C_{i}$ such that $a, b \in C_{i}$ for some $\{a, b\} \in E$.

Proof of Theorem 1. Let $(X, E)$ be such a graph for $m=2^{n}$. Let $(X, R)$ be any digraph for which $x y \in R$ or $y x \in R$ if and only if $\{x, y\} \in E$. Suppose that $R$ is the union of partial orders $P_{1}, \cdots, P_{n}$ on $X$. Since $E$ has no triangles, any subset of a $P_{i}$ is a partial order and hence we can assume $P_{i} \cap P_{j}=\varnothing$ when $i \neq j$. Letting $A(x)=\{i$ : for some $\left.y \in X, x y \in P_{i}\right\}$, partition $X$ so that $x$ and $y$ are in the same element of the partition if and only if $A(x)=A(y)$. The number of elements in the partition does not exceed $2^{n}$. Thus, by Lemma 1 , the partition contains an element $Y$ with $x, y \in Y$ and $\{x, y\} \in E$. Then $A(x)=$ $A(y)$. Since $x y \in R$ or $y x \in R$, take $x y \in P_{j}$ for definiteness with $j \in A(x)$. Since $j \in A(y)$ also, there is a $z \in X$ such that $y z \in P_{j}$. Transitivity then implies that $x z \in P_{j}$ and hence that $E$ includes a triangle, which contradicts our initial hypothesis. Therefore $\sigma(R)>n$. By the definition of $R$ it can be taken to be either asymmetric or symmetric (or neither).

Henceforth in this section all logarithms are to base 2 unless indicated otherwise. $[r]=$ (largest integer $\leqq r$ ) and $\{r\}=$ (smallest integer $\geqq r$ ).

THEOREM $2 . \quad \log n-1 / 2 \log \log n+0(1) \geqq s(n) \geqq \log n-3 / 2 \log \log n$ $-0(1)$.

We show first the upper bound, using two preparatory lemmas. 
Lemma 2. In any digraph $G=(H, R)$ with $|H|=m$ there exists $D \subseteq H$ such that $|D| \geqq\left\{\log _{4} m\right\}=\{1 / 2 \log m)$ and $\sigma(G \mid D) \leqq 2$.

Proof. We use induction on $m$, the lemma being obvious for small values of $m$. Fix $x \in H$. Split $H^{*}=H-\{x\}$ into four parts:

$$
\begin{array}{ll}
T_{1}=\left\{y \in H^{*}: x y \notin R \& y x \notin R\right\} & S_{1}=\varnothing \\
T_{2}=\left\{y \in H^{*}: x y \in R \& y x \notin R\right\} & S_{2}=\{x\} \times D_{2} \\
T_{3}=\left\{y \in H^{*}: x y \notin R \& y x \in R\right\} & S_{3}=D_{3} \times\{x\} \\
T_{4}=\left\{y \in H^{*}: x y \in R \& y x \in R\right\} & S_{4}^{\prime}=\{x\} \times D_{4}, \\
& S_{4}^{\prime \prime}=D_{4} \times\{x\} .
\end{array}
$$

Some $\left|T_{i}\right| \geqq\{(m-1) / 4\}$. By induction find $D_{i} \subseteq T_{i}$ with

$$
\left|D_{i}\right| \geqq\left\{\log _{4}\left|T_{i}\right|\right\} \geqq\left\{\log _{4}\{(m-1) / 4\}\right\}=\left\{\log _{4} m\right\}-1
$$

and $G \mid D_{i}=P_{1} \cup P_{2}$. Then set $D=D_{i} \cup\{x\} . G \mid D=\left(P_{1} \cup S_{i}\right) \cup\left(P_{2} \cup S_{i}\right)$ except for $i=4$ when $G \mid D=\left(P_{1} \cup S_{4}^{\prime}\right) \cup\left(P_{2} \cup S_{4}^{\prime \prime}\right)$.

Lemma 3. In any digraph $G=(X, R)$ with $|X|=n$ there is a partition $\left\{D_{1}, \cdots, D_{t}\right\}$ of $X$ such that $t<3 n / \log n$ and $\sigma\left(G \mid D_{i}\right) \leqq 2$ for each $i$.

Proof. Given $G$, by Lemma 2 find $D_{1}$ such that

$$
\left|D_{1}\right|=x_{1} \geqq\left\{\log _{4} n\right\} \text {. }
$$

By induction find $D_{i}$ such that

$$
\left|D_{i}\right|=x_{i} \geqq\left\{\log _{4}\left(n-\sum_{j=1}^{i-1} x_{j}\right)\right\} \text {. }
$$

From elementary calculus we can show $\sum_{i=1}^{t} x_{i} \geqq n$ for

$$
t \leqq(2+\varepsilon) n / \log n \text {. }
$$

We now show the upper bound for Theorem 2. Let $G=(X, R)$ with $|X|=n$. Take $D_{1}, \cdots, D_{t}$ as in Lemma 3. Let $\left\{A_{i}^{*}, B_{i}^{*}\right\}$ be a partition of $\{1, \cdots, t\}$ for $i=1, \cdots, s$ such that for all $1 \leqq j \neq k \leqq t$ there exists $i, 1 \leqq i \leqq s$, such that $j \in A_{i}^{*} \& k \in B_{i}^{*}$. By Spencer [12] we may take

$$
s=\log t+1 / 2 \log \log t+0(1) \leqq \log n-1 / 2 \log \log n+0(1) .
$$

$\left\{A_{i}^{*}, B_{i}^{*}\right\}$ induces a partition $\left\{A_{i}, B_{i}\right\}$ of $X$ with

$$
A_{i}=\bigcup_{j \in A_{i}^{*}} D_{j}, \quad B_{i}=\bigcup_{j \in B_{i}^{*}} D_{j}
$$


Then set

$$
P_{i}=\left\{x y: x \in A_{i} \& y \in B_{i} \& x y \in R\right\} \quad \text { for } i=1, \cdots, s .
$$

Since $\sigma\left(G \mid D_{i}\right) \leqq 2, G \mid D_{i}=P_{i}^{\prime} \cup P_{i}^{\prime \prime}$. Set

$$
P^{\prime}=\bigcup_{i=1}^{s} P_{i}^{\prime}, P^{\prime \prime}=\bigcup_{i=1}^{s} P_{i}^{\prime \prime} .
$$

Then $R=P^{\prime} \cup P^{\prime \prime} \cup P_{1} \cup \cdots \cup P_{s}$, giving the upper bound of Theorem 2.

We turn to the lower bound of the theorem, again using two preliminary lemmas. A complete asymmetric digraph is a tournament. ${ }^{4}$ We shall show that a "random" tournament $T=(X, R)$ with $|X|=n$ has $\sigma(T) \geqq \log n-3 / 2 \log \log n-0(1)$. Intuitively speaking, we show that all $P \subseteq T$ are essentially bipartite.

Let $T^{n}$ be the set of tournaments with $X=\{1,2, \cdots, n\}$. We say that $T=(X, R) \in T^{n}$ has property $\alpha$ if and only if there are $A$, $B \subseteq X$ with $|A|=|B| \geqq 3 \log n$ and $A \times B \subseteq R$. T has property $\beta$ if and only if there is an $A \subseteq X$ and a linear order $L$ on $A$ such that $|A| \geqq(\log n)^{2}$ and

$$
|R \cap L| \leqq \frac{1}{3}\left(\begin{array}{c}
|A| \\
2
\end{array}\right)
$$

Lemma 4. For $n$ sufficiently large there exists $T \in T^{n}$ satisfying neither property $\alpha$ nor property $\beta$.

Proof. If $T \in T^{n}$ has property $\alpha$, there are $A, B \cong X$ with $|A|=|B|=[3 \log n]$ and $A \times B \subseteq R$. Set $t=[3 \log n]$. For fixed $A$ and $B, 2^{-t^{2}}$ is the proportion of $T \in T^{n}$ that satisfy this condition. There are less than $n^{2 t}$ choices of $A$ and $B$, so less than $n^{2 t} 2^{-t^{2}}$ of the $T \in T^{n}$ satisfy $\alpha . \quad n^{2 t} 2^{-t^{2}} \rightarrow 0$ as $n \rightarrow \infty$.

If $T \in T^{n}$ has property $\beta$, there exists $A \subseteq X$ and $L$ on $A$ such that $|A|=\left[(\log n)^{2}\right]$ and $\left(^{*}\right)$ holds. There are less than $n^{(\log n)^{2}}$ choices of $A$ and then $\left[(\log n)^{2}\right]$ ! choices of $L$. Given $A$ and $L$, the proportion of $T \in T^{n}$ satisfying $\left(^{*}\right)$ is the probability of at most $\left(\begin{array}{l}t \\ 2\end{array}\right) / 3$ heads in ( $\left.\begin{array}{l}t \\ 2\end{array}\right)$ flips of a fair coin where $t=|A| \sim(\log n)^{2}$. This probability is approximately $p^{-\left(\frac{t}{2}\right)}$ where $p=3^{1 / 3}(3 / 2)^{2 / 3}>1$. Thus the proportion of $T \in T^{n}$ satisfying $\beta$ is less than

$$
n^{(\log n)^{2}}\left[(\log n)^{2}\right] ! p^{-\left(\frac{t}{2}\right)}, \text { which } \longrightarrow 0 \text { as } n \longrightarrow \infty .
$$

Thus for $n$ sufficiently large some $T \in T^{n}$ can satisfy neither $\alpha$ nor $\beta$.

${ }^{4}$ See Moon [9] for extensive discussion of tournaments. See also $[3,10,11]$ for resulted to the present paper. 
Lemma 5. If $T_{1}, \cdots, T_{n} \subseteq\{1, \cdots, s\}$ then there are $n /\left({ }_{s / 2}^{s}\right) \quad T_{i}$ which are mutually comparable. ${ }^{6}$

Proof. We use a technique due to Lubell [8]. There are $s$ ! maximal chains of subsets of $\{1, \cdots, s\}$ under the ordering of $\subset$. If $\left|T_{i}\right|=\mathrm{a}$ then $T_{i}$ is in $a !(s-a) ! \geqq(s / 2) !^{2}=s ! /\left(\begin{array}{c}s \\ s / 2\end{array}\right)$ maximal chains. Thus some maximal chain must contain $n\left[s ! /\left({ }_{s / 2}^{s}\right)\right] / s ! T_{i}$.

In the following proof of the lower bound of Theorem 2 we use the fact that $1 /\left(\begin{array}{c}s / 2 \\ s / 2\end{array}\right) \sim \sqrt{\pi / 2} \sqrt{s} 2^{-s}$.

Let $G=(X, R)$ be a tournament that satisfies neither $\alpha$ nor $\beta$ (Lemma 4). Suppose that $R=P_{1} \cup \cdots \cup P_{s}$. Define

$$
\begin{aligned}
& W_{i}=\left\{x \in X:\left|\left\{y \in X: x y \in P_{i}\right\}\right|>3 \log n\right\} \\
& L_{i}=\left\{x \in X:\left|\left\{y \in X: y x \in P_{i}\right\}\right|>3 \log n\right\} \\
& R_{i}=X-W_{i}-L_{i}
\end{aligned}
$$

for $1 \leqq i \leqq s$. (We split $X$ into winners, losers, and the rest.) By Lemma $4, W_{i} \cap L_{i}=\varnothing$. For $x \in X$ set

$$
T_{x}=\left\{i: x \in W_{i} \cup R_{i}\right\} \subseteq\{1, \cdots, s\} .
$$

By Lemma 5 find $V \subseteq X$ such that $|V| \geqq n \sqrt{\pi / 2} \sqrt{s} 2^{-s}$ and $T_{x} \subseteq T_{y}$ or $T_{y} \subseteq T_{x}$ whenever $x, y \in V$. Induce a linear order $L$ on $V$ by setting $x y \in L$ if $T_{x} \subset T_{y}$ : when $T_{x}=T_{y}, L$ is defined in any fixed manner.

Now assume $s<\log n-3 / 2 \log \log n-7$. Then $|V| \geqq 2^{7} \sqrt{\pi / 2}$ $(\log n)^{2}$. Set

$$
Z_{i}=L \cap P_{i} \quad 1 \leqq i \leqq s .
$$

Given $x y \in Z_{i}, \quad T_{x} \subseteq T_{y}$ so that we cannot have $x \in W_{i} \& y \in L_{i}$. And since $W_{i} \cap L_{i}=\varnothing$ we cannot have $x \in L_{i} \& y \in W_{i}$. Therefore

$$
Z_{i}=\left\{x y \in Z_{i}: x \text { or } y \in R_{i}\right\} \cup\left\{x y \in Z_{i}: x, y \in W_{i}\right\} \cup\left\{x y \in Z_{i}: x, y \in L_{i}\right\} \text {. }
$$

There are at most $6 \log n|V|, 3 \log n|V|$ and $3 \log n|V|$ ordered pairs in the first, second and third parts respectively of this decomposition of $Z_{i}$. Thus $\left|Z_{i}\right| \leqq 12 \log n|V|$. Since $G$ does not have property $\beta$ it follows that

$$
\frac{1}{3}\left(\begin{array}{c}
|V| \\
2
\end{array}\right) \leqq|R \cap L| \leqq \sum_{i=1}^{n}\left|Z_{i}\right| \leqq 12(\log n)^{2}|V|
$$

and hence that $|V| \leqq 72(\log n)^{2}+1$. Since this contradicts $|V| \geqq 2^{7}$ $\sqrt{\pi / 2}(\log n)^{2}$ it must be true that $s \geqq \log n-3 / 2 \log \log n-0(1)$. 
This completes the proof of Theorem 2.

If a sufficiently good bound could be placed on

$$
\left\{x y \in P_{i}: x \text { or } y \in R_{i} \text { or } x, y \in W_{i} \text { or } x, y \in L_{i}\right\}
$$

then one could prove $s(n)=\log n-1 / 2 \log \log n+o(\log \log n)$. One might even show that $s(n)=\log n-1 / 2 \log \log n+0(1)$.

3. A digraph with $\sigma=4$ and $|X|=13$. Although the theorems of the preceding section show that there are digraphs with large indices, they are of little use in attempting to discover the smallest $X$ that admits an $R$ for which $\sigma(R)=n$. Figure 1 shows the smallest $X$ that we know of for which $\sigma(R)=4$.

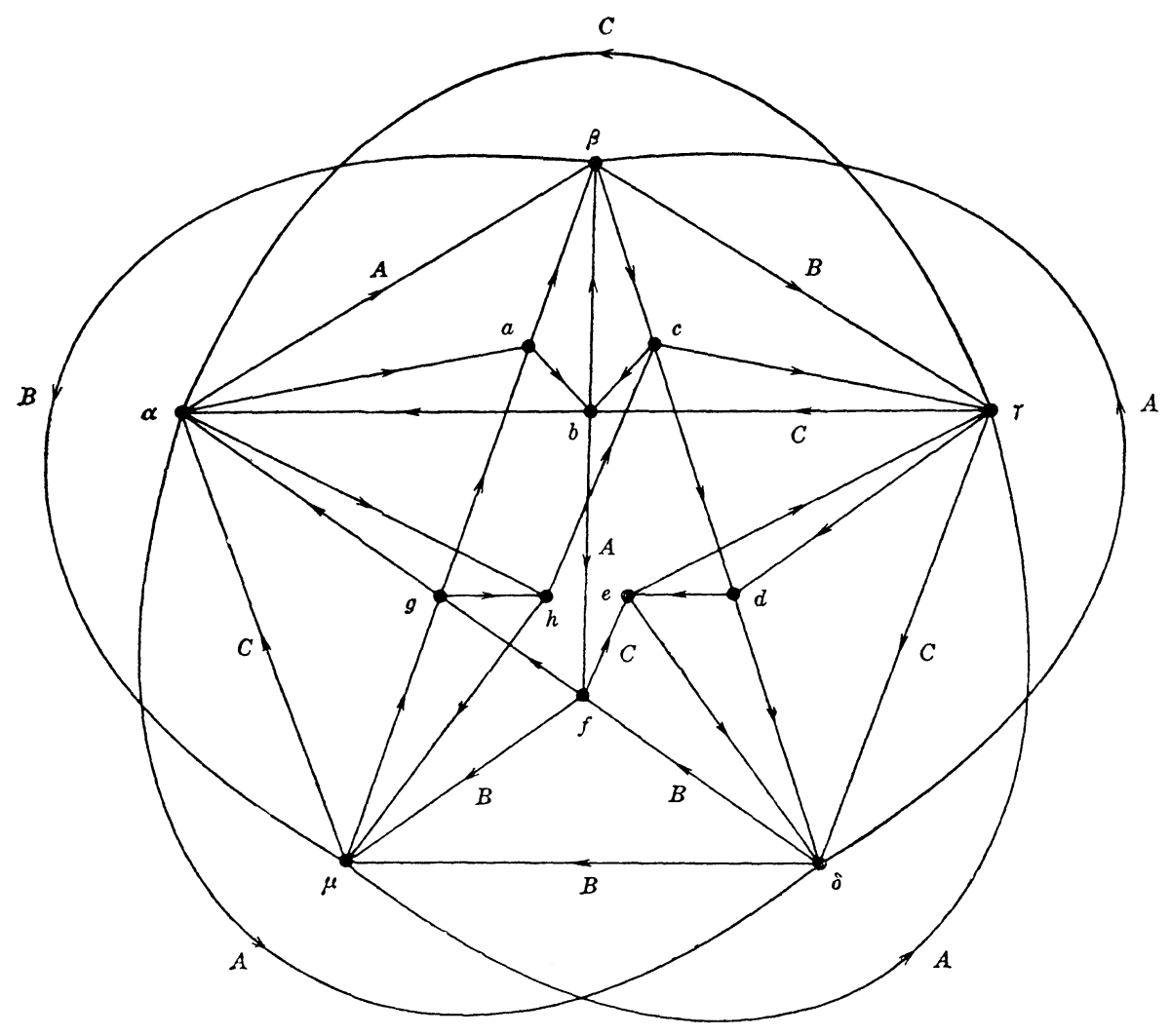

Figure 1

Assume that $\sigma(R)=3$ for Figure 1, with $A, B$ and $C$ three partial orders whose union equals $R$. Then one of $A, B$ and $C$ must contain exactly one of $\alpha \beta, \beta \gamma, \gamma \delta, \delta \mu$ and $\mu \alpha$ and the other two must each contain exactly two of these ordered pairs in alternating fashion. 
Suppose for example that $\alpha \beta \in A, \beta \gamma \in B, \gamma \delta \in C, \delta \mu \in B, \mu \alpha \in C$. Then $\gamma \alpha, \delta \beta, \mu \gamma, \alpha \delta$, and $\beta \mu$ must be respectively in $C, A, A, A$, and $B$. Then $\gamma b \in C$ and $\delta f, f \mu \in B$. Since $\gamma b \in C$ and $f \mu \in B, b f \in A$. Since $b f \in A$ and $\delta f \in B, f e \in C$. By the cyclic triangle $\{f e, e \delta, \delta f\}, e \delta$ must be in $A$. But since $\delta \beta \in A$ this implies $e \beta \in A$, which is false. A similar contradiction to $\sigma=3$ is obtained when any alternative assignment is made for $a \beta, \beta \gamma, \cdots, \mu \alpha$.

4. Indices of symmetric digraphs. In this section we consider symmetric $(x y \in S \Rightarrow y x \in S)$ digraphs $(X, S)$. For any binary relation $R, R^{*}=\{x y: y x \in R\}$, the converse or dual of $R$.

A graph $(X, E)$ is a comparability graph if and only if there is a partial order $P$ on $X$ such that $\{x, y\} \in E$ if and only if $x y \in P \cup P^{*}$. Ghouila-Houri [5] and Gilmore and Hoffman [6] provide characterizations of comparability graphs. When $(X, S)$ is a symmetric digraph, $(X, E(S))$ will denote the graph in which $\{x, y\} \in E(S)$ if and only if $x y \in S$.

THEOREM 3. Suppose that $(X, S)$ is a symmetric digraph. Then $\sigma(S) \leqq 2$ if and only if $(X, E(S))$ is a comparability graph.

Proof. If $(X, E(S))$ is a comparability graph then $S=P \cup P^{*}$ for a partial order $P$, and thus $\sigma(S) \leqq 2$. Conversely, if $S=P_{1} \cup P_{2}$ with $P_{1}$ and $P_{2}$ partial orders, then $P_{2}=P_{1}^{*}$.

In [1] it is shown that if $(X, P)$ is a transitive digraph (so that $P$ is a partial order) and if $S=\left\{x y: x \neq y \& x y \notin P \cup P^{*}\right\}$ then $D(P) \leqq 2$ if and only if $(X, E(S))$ is a comparability graph. Hence, as a corollary to Theorem 3 we have $D(P) \leqq 2$ if and only if $\sigma(S) \leqq 2$. Our next theorem extends this in one direction.

Theorem 4. Suppose that $P$ on $X$ is a partial order and let $S=\left\{x y: x \neq y\right.$ \& $\left.x y \notin P \cup P^{*}\right\}$. Then $D(P) \leqq n \Rightarrow \sigma(S) \leqq 2(n-1)$ for $n>1$.

Proof. The theorem is true for $n=2$. Using induction, assume it's true for all $n<m$ and suppose $D(P)=m$ with $P=\bigcap_{1}^{m} L_{i}$ where each $L_{i}$ is a linear order. Let $P^{\prime}=\bigcap_{2}^{m} L_{i}$ and

$$
S^{\prime}=\left\{x y: x \neq y \& x y \notin P^{\prime} \cup\left(P^{\prime}\right)^{*}\right\} \text {. }
$$

Since $D\left(P^{\prime}\right) \leqq m-1$, the induction hypothesis gives $\sigma\left(S^{\prime}\right) \leqq 2(m-2)$. Clearly $S^{\prime} \subseteq S$ and $S-S^{\prime}=\left(P^{\prime} \cap L_{1}^{*}\right) \cup\left(\left(P^{\prime}\right)^{*} \cap L_{1}\right)$. Since $P^{\prime} \cap L_{1}^{*}$ is a partial order (the intersection of two partial orders) and $\left(P^{\prime}\right)^{*} \cap L_{1}$ is a partial order, $\sigma(S) \leqq \sigma\left(S^{\prime}\right)+2 \leqq 2(m-2)+2=2(m-1)$. 
5. Almost transitive digraphs. The proof of the next theorem has several similarities to Szpilrajn's proof [13] of the theorem that any partial order $P$ on $X$ can be extended to a linear order $L$ with $P \subseteq L$. We recall that $R$ is almost transitive if and only if ( $a b \in R$ $\& b c \in R \& a \neq c) \Rightarrow a c \in R$.

THEOREM 5. $\sigma(R) \leqq 2$ if $(X, R)$ is an almost transitive digraph.

Proof. Assume that $(X, R)$ is an almost transitive digraph. Let $A=\{a b: a b \in R \& b a \notin R\}$, the asymmetric part of $R$. Let $A^{+}=$ $\left\{a b: a b \in A\right.$ or $\left\{a a_{1}, a_{1} a_{2}, \cdots, a_{n} b\right\} \subseteq A$ for distinct $a_{1}, \cdots, a_{n}$ in $X$ that are different from $a$ and $b$, the almost transitive closure of $A$. Clearly $A^{+} \subseteq R$ and $A^{+}$is almost transitive.

To show that $A^{+}$is a partial order it suffices to show that it is asymmetric. To the contrary suppose that $x y \in A^{+}$and $y x \in A^{+}$. Then from the definition of $A^{+}$and almost transitivity for $R$ it follows easily that there is a $c \in X$ for which $c x \in A$ and $x c \in R$, which contradicts the definition of $A$. Hence $A^{+}$is a partial order.

Let $\mathscr{P}=\left\{P: P\right.$ is a partial order on $\left.X \& A^{+} \subseteq P \subseteq R\right\}$. It follows easily from Zorn's lemma that there is a $P^{*} \in \mathscr{P}^{\circ}$ such that $P^{*} \subset P$ for no $P \in \mathscr{P}$. Letting $P^{*}$ be maximal in this sense we now prove that

$$
a b, b a \in R \Rightarrow a b \in P^{*} \text { or } b a \in P^{*} .
$$

To the contrary suppose that each of $a b$ and $b a$ is in $R$ and neither is in $P^{*}$. Then let

$$
W=\left\{x y: x \neq y \&\left(x a \in P^{*} \text { or } x=a\right) \&\left(b y \in P^{*} \text { or } y=b\right)\right\},
$$

and let $V=P^{*} \cup W$, so that $P^{*} \subset V$. We show that $V$ is a partial order (clearly $A^{+} \subseteq V \subseteq R$ ), thus contradicting the maximality of $P^{*}$. $V$ is irreflexive since $P^{*}$ and $W$ are irreflexive. For transitivity take $x y, y z \in V$. If both $x y$ and $y z$ are in $P^{*}$ then $x z \in P^{*}$ by the transitivity of $P^{*}$.

Suppose next that $x y \in P^{*}$ and $y z \in W$. The latter gives $\left(y a \in P^{*}\right.$ or $y=a)$, from which $x a \in P^{*}$ follows, and it gives also $\left(b z \in P^{*}\right.$ or $z=b$ ), from which $x z \in V$ follows unless $x=z$. But if $x=z$ we have $x a \in P^{*}$ and $\left(b x \in P^{*}\right.$ or $x=b$ ), which give $b a \in P^{*}$, contradicting the hypothesis that $b a \notin P^{*}$. Hence $x y \in P^{*} \& y z \in W \Rightarrow x z \in V$. Similarly, $x y \in W \& y z \in P^{*} \Rightarrow x z \in V$.

The final case for transitivity is $x y, y z \in W$. Then $\left(x a \in P^{*}\right.$ or $x=a)$ and $\left(b z \in P^{*}\right.$ or $\left.z=b\right)$ so that $x z \in W$ unless $x=z$. But if $x=z$ then $\left[\left(x a \in P^{*}\right.\right.$ or $\left.x=a\right) \&\left(b x \in P^{*}\right.$ or $\left.\left.x=b\right)\right] \Rightarrow\left(b a \in P^{*}\right.$ or $\left.b=a\right)$, which is false. Hence $V$ is a partial order, a contradiction to the 
maximality of $P^{*}$, and therefore

$$
a b, b a \in R \Longrightarrow a b \in P^{*} \text { or } b a \in P^{*} \text {. }
$$

Finally, let $Q=R-P^{*}$ so that $R=P^{*} \cup Q . \quad Q$ is irreflexive since $R$ is irreflexive. Suppose that $x y, y z \in Q$. Then, since both $x y$ and $y z$ are in $R$ but not $A, y x$ and $z y$ are in $R$ and must be in $P^{*}$ by the preceding analysis. Therefore $z x \in P^{*}$ and $z \neq x$. Then, by almost transitivity of $R, x z \in R$ and thus $x z \in Q$ since $P^{*}$ is asymmetric.

Thus $R=P^{*} \cup Q$, the union of two partial orders.

6. A partition characterization for $\sigma \leqq 2$. Given a digraph $(X, R)$ let $K$ be the set of all ordered pairs of pairs in $R$ that deny transitivity, so that

$x y K y z$ if and only if $x y \in R \& y z \in R \& x z \notin R$,

and let $V$ be the subset of $R$ involved in these intransitivities so that

$$
V=\{x y: x y K y z \text { or } z x K x y \text { for some } z \in X\} \text {. }
$$

Suppose that $\sigma(R) \leqq 2$. If $x y K y z$ then $x y$ and $y z$ must be in different resolving partial orders, so that the digraph $(V, K)$ must be bipartite or 2-colorable. Moreover, if $x y$ and $y z$ are in $V$ and in the same resolving partial order and if $x z \in V$ also, then transitivity requires that $x z$ be in this partial order. These two necessary conditions for $\sigma(R) \leqq 2$ are reflected in $\mathrm{A} 1$ and $\mathrm{A} 2$ of Theorem 6 . Their insufficiency for $\sigma(R) \leqq 2$ is noted later. (Note that $\sigma(R)=1$ if and only if $V=\varnothing$.)

Theorem 6. Suppose that $(X, R)$ is a digraph and $V \neq \varnothing$. Then $\sigma(R)=2$ if and only if $V$ can be partitioned into $V_{1}$ and $V_{2}$ so that

A1. $x y K y z \Rightarrow x y$ and $y z$ are in different $V_{i}$,

A2. $x y, y z \in V_{i} \& x z \in V \Rightarrow x z \in V_{i}$,

A3. $x y \in R-V \Rightarrow(1)$ and (2) do not hold simultaneously:

$$
\left(y z \in V_{2} \& x z \in V_{1}\right) \text { or }\left(z x \in V_{2} \& z y \in V_{1}\right) \text {, for some } z \in X \text {, }
$$

$$
\left(y w \in V_{1} \& x w \in V_{2}\right) \text { or }\left(w x \in V_{1} \& w y \in V_{2}\right) \text {, for some } w \in X \text {. }
$$

If $R=P_{1} \cup P_{2}$ then $V_{i}=P_{i} \cap V$ for $i=1,2$ are easily seen to satisfy $\mathrm{A} 1$ through $\mathrm{A} 3$, and $V_{1} \cap V_{2}=\varnothing$.

Before proving sufficiency we show that A1 and A2 are not sufficient for $\sigma=2$. All directed edges in the 13-point asymmetric 


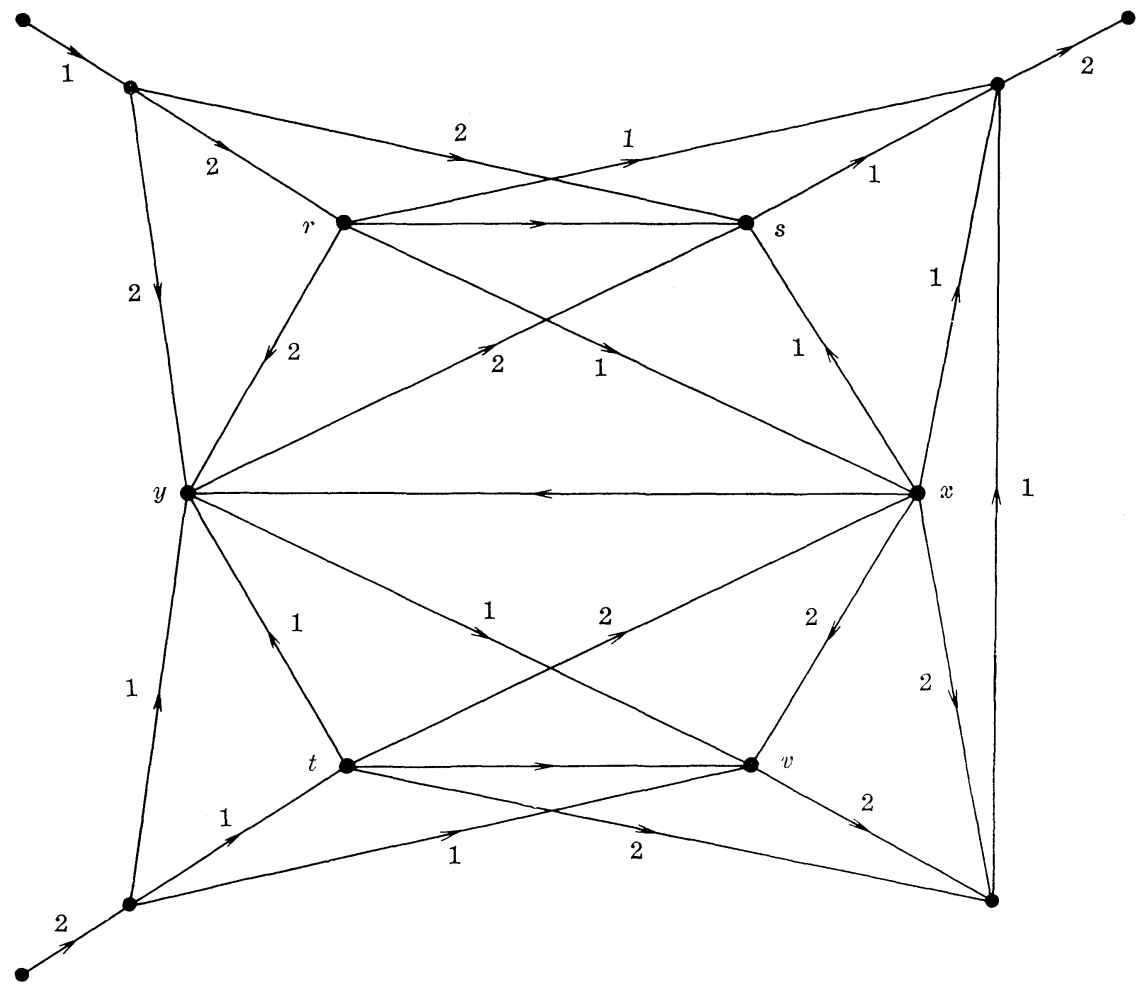

FIGURE 2

digraph of Figure 2 are in $V$ except for $x y, r s$ and $t v$, and $\mathrm{A} 1$ and A2 hold. Labels 1 and 2 for $P_{1}$ and $P_{2}$ are assigned to the edges in $V$ in the only way consistent with $\mathrm{A} 1$ and A2, beginning with $P_{1}$ in the upper left corner. For $\sigma(R)=2$ we require $r s$ and $t v$ in both $P_{1}$ and $P_{2}$, but $x y$ violates $\mathrm{A} 3$ and cannot be assigned either

$$
P_{1}\left[r x \in P_{1} \& r y \notin P_{1}\right] \text { or } P_{2}\left[t x \in P_{2} \& t y \notin P_{2}\right] \text {. }
$$

By deleting the edge $x y$ from Figure 2 we obtain an $R$ with $\sigma(R)=2$ where $R$ is not the union of two disjoint partial orders.

Sufficiency Proof for Theorem 6. With $V \neq \varnothing$ let A1, A2 and A3 hold. For $i=1,2$ let

$$
S_{i}=\{x y: x y \in R-V \& \text { (i) holds }\} \text {. }
$$

Let $R^{0}=R-V-S_{1}-S_{2}$ and for $i=1,2$ define $P_{i}$ by

$$
P_{i}=V_{i} \cup S_{i} \cup R^{0} \text {. }
$$

Since $P_{i} \subseteq R$, it is irreflexive. We now prove that $P_{1}$ is transitive. The proof for $P_{2}$ is similar. 
Assume that $x y, y z \in P_{1}$. Then $x z \in R$, for if both $x y$ and $y z$ are in $V_{1}$ then $x z \in R$ by $\mathrm{A} 1$, and if one of $x y$ and $y z$ is in $S_{1} \cup R^{0}$ then $x z \in R$ by the definitions. Thus $x z \in P_{1}$ unless $x z \in V_{2} \cup S_{2} . \quad x z \in V_{2}$ is contradicted in all cases:

1. $x y, y z \in V_{1} \Rightarrow x z \notin V_{2}$, by $\mathrm{A} 2$;

2. $x y \in V_{1} \& y z \in S_{1} \Rightarrow x z \notin V_{2}$, by A3;

3. $x y \in V_{1} \& y z \in R^{0} \Rightarrow x z \notin V_{2}$, by A3;

4. $x y, y z \in S_{1} \cup R^{0}$. Then $a x \in R \Rightarrow a y \in R \Rightarrow a z \in R \quad$ and $z a \in R \Rightarrow y a \in R \Longrightarrow x a \in R$. Hence neither $a x K x z$ nor $x z K z a$ can hold. It remains to show that $x z \notin S_{2}$. Assume $x z \in S_{2}$ to the contrary and for definiteness take $z w \in V_{1}$ and $x w \in V_{2}$ (Figure 3). We note first

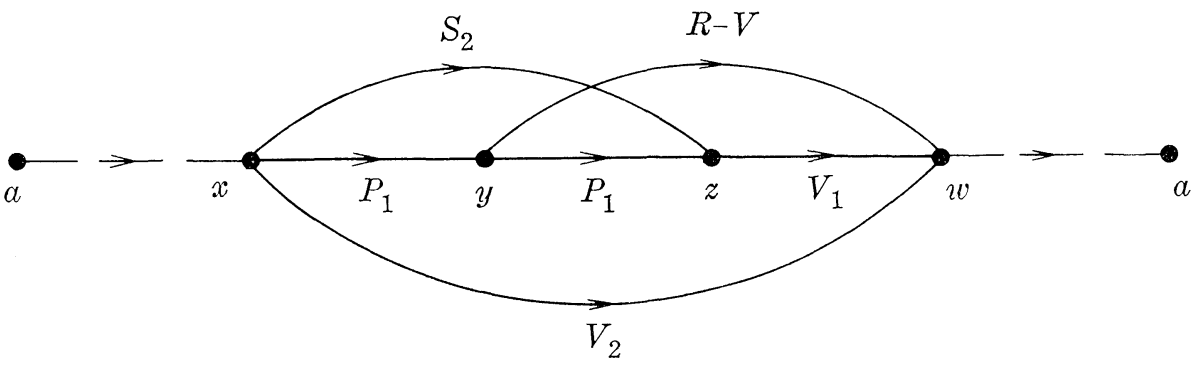

FiguRE 3

that $y w \notin V_{2}$, for $y w \in V_{2} \Rightarrow y z \in S_{2}$. Moreover, $y w \notin V_{1}$, for $y w \in V_{1}$ $\& x y \in V_{1}$ contradict $\mathrm{A} 2$, and $y w \in V_{1} \& x y \in S_{1} \cup R^{0}$ contradict the definition of $S_{2}$ along with A3. Hence $y w \in R-V$. Now if $a x \in V_{1}$ then $a y \in R$ and hence (since $y w \in R-V$ ) $a w \in R$; and if $w a \in V_{1}$ then $z a \in R$ and hence (since $x z \in R-V$ ) $x a \in R$. Since $x w \in V_{2}$ requires either $a x K x w$ with $a x \in V_{1}$ or $x w K w a$ with $w a \in V_{1}$, and since $a x \in V_{1}$ contradicts $a x K x w$ (since $a w \in R$ ) and $w a \in V_{1}$ contradicts $x w K w a$ (since $x a \in R$ ), the proof is complete.

\section{REFERENCES}

1. K. A. Baker, P. C. Fishburn, and F. S. Roberts, Partial Orders of Dimension 2, Interval Orders, and Interval Graphs, RAND Paper P-4376, The RAND Corporation, 1970.

2. B. Dushnik and E. W. Miller, Partially Ordered Sets, Amer. J. Math., 63 (1941), 600-610.

3. P. Erdös and L. Moser, On the Representation of Directed Graphs as Unions of Orderings, Math. Inst. Hung. Acad. Sci., 9 (1964), 125-132.

4. J. Folkman, Graphs with Monochromatic Complete Subgraphs in Every Edge Coloring, SIAM J. Appl. Math., 18 (1970), 19-24.

5. A. Ghouila-Houri, Caractérisation des graphes nonorientés dont on peut orienter les arêtes de manière à obtenir le graphe d'une relation d'ordre, C. R. Acad. Sci. Paris 254 (1962), 1370-1371. 
6. P. C. Gilmore and A. J. Hoffman, A Characterization of Comparability Graphs and of Interval Graphs, Canad. J. Math., 16 (1964), 539-548.

7. F. Harary, R. Z. Norman, and D. Cartwright, Structural Models: An Introduction to the Theory of Directed Graphs, Wiley, New York, 1965.

8. D. Lubell, A Short Proof of Sperner's Lemma, J. Combinatorial Theory, 1 (1966), 299.

9. J. W. Moon, Topics on Tournaments, Holt, New York, 1969.

10. K. B. Reid and E. T. Parker, Disproof of a conjecture of Erdös and Moser on tournaments, J. Combinatorial Theory, 9 (1970), 225-238..

11. J. Spencer, Notes on Combinatorial Mathematics: Transitive Subtournaments, RAND Memorandum RM-6156-PR, The RAND Corporation, 1969.

12. - Minimal Completely Separating Systems, J. Combinatorial Theory, 8 (1970), 446-447.

13. E. Szpilrajn, Sur extension de l'ordre partiel. Fundamenta Math., 16 (1930), 386-389.

Received July 22, 1970 and in revised form October 12, 1970.

The Institute for Advanced Study and the Research Analysis Corporation AND

The RAND Corporation, Santa Monica 



\section{PACIFIC JOURNAL OF MATHEMATICS}

\section{EDITORS}

H. SAMELSON

Stanford University

Stanford, California 94305

C. R. HobBY

University of Washington

Seattle, Washington 98105
J. DugundjI

Department of Mathematics

University of Southern California

Los Angeles, California 90007

RICHARD ARENS

University of California

Los Angeles, California 90024

\section{ASSOCIATE EDITORS}

E. F. BECKENBACH

B. H. NeUMaNN

F. WOLF

K. YoshidA

\section{SUPPORTING INSTITUTIONS}

UNIVERSITY OF BRITISH COLUMBIA

CALIFORNIA INSTITUTE OF TECHNOLOGY

UNIVERSITY OF CALIFORNIA

MONTANA STATE UNIVERSITY

UNIVERSITY OF NEVADA

NEW MEXICO STATE UNIVERSITY

OREGON STATE UNIVERSITY

UNIVERSITY OF OREGON

OSAKA UNIVERSITY
UNIVERSITY OF SOUTHERN CALIFORNIA STANFORD UNIVERSITY

UNIVERSITY OF TOKYO

UNIVERSITY OF UTAH

WASHINGTON STATE UNIVERSITY UNIVERSITY OF WASHINGTON

$\stackrel{*}{*} \stackrel{*}{*} \stackrel{*}{*}$ AMERICAN MATHEMATICAL SOCIETY
NAVAL WEAPONS CENTER 


\section{Pacific Journal of Mathematics}

Vol. 39 , No. 1

May, 1971

Charles A. Akemann, A Gelfand representation theory for $C^{*}$-algebras ....

Sorrell Berman, Spectral theory for a first-order symmetric system of

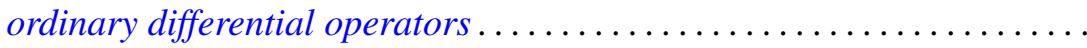

Robert L. Bernhardt, III, On splitting in hereditary torsion theories ........

J. L. Brenner, Geršgorin theorems, regularity theorems, and bounds for determinants of partitioned matrices. II. Some determinantal identities ..........................................

Robert Morgan Brooks, On representing $F^{*}$-algebras .............. 51

Lawrence Gerald Brown, Extensions of topological groups........... 71

Arnold Barry Calica, Reversible homeomorphisms of the real line ........ 79

J. T. Chambers and Shinnosuke Oharu, Semi-groups of local Lipschitzians in

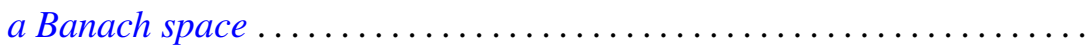

Thomas J. Cheatham, Finite dimensional torsion free rings .............

Byron C. Drachman and David Paul Kraines, A duality between

transpotence elements and Massey products ...................

Richard D. Duncan, Integral representation of excessive functions of a

Markov process ......................................

George A. Elliott, An extension of some results of Takesaki in the reduction

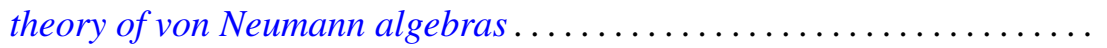

Peter C. Fishburn and Joel Spencer, Directed graphs as unions of partial

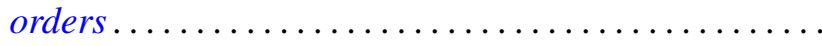

Howard Edwin Gorman, Zero divisors in differential rings ...

Maurice Heins, A note on the Löwner differential equations...

Louis Melvin Herman, Semi-orthogonality in Rickart rings. .

David Jacobson and Kenneth S. Williams, On the solution of linear G.C.D.

equations

Michael Joseph Kallaher, On rank 3 projective planes ... . .

Donald Paul Minassian, On solvable $O^{*}$-groups ...........

Nils Øvrelid, Generators of the maximal ideals of $A(\bar{D})$

Mohan S. Putcha and Julian Weissglass, A semilattice decomposition into

semigroups having at most one idempotent ............

Robert Raphael, Rings of quotients and $\pi$-regularity ....

J. A. Siddiqi, Infinite matrices summing every almost periodic sequence. .

Raymond Earl Smithson, Uniform convergence for multifunctions ...

Thomas Paul Whaley, Mulitplicity type and congruence relations in

universal algebras...

Roger Allen Wiegand, Globalization theorems for locally finitely generated modules... 